\title{
Semi-dynamic Hawk and Dove game, applied to power control
}

\author{
Eitan Altman*, Dieter Fiems ${ }^{\dagger}$, Majed Haddad*, and Julien Gaillard* \\ *INRIA Sophia Antipolis, 10 route des Lucioles, 06902 Sophia Antipolis, France \\ ${ }^{\dagger}$ SMACS Research Group, Department TELIN, Ghent University, Belgium
}

\begin{abstract}
In this paper, we study a power control game over a collision channel. Each player has an energy state. When choosing a higher transmission power, the chance of a successful transmission (in the presence of other interference) increases at the cost of a larger decrease in the energy state of the battery. We study this dynamic game when restricting to simple nondynamic strategies that consist of choosing a given power level that is maintained during the lifetime of the battery. We identify a surprising paradox in our Hawk-Dove game which we term the initial energy paradox.
\end{abstract}

\section{INTRODUCTION}

This paper investigates a semi-dynamic variant of the well known Hawk and Dove game [3]. The latter game has been introduced to describe the evolution of aggressive behavior among animals competing for food. Variants of the Hawk and Dove (HD) game have found various applications in networking. The medium access game considers competition over the access to a common channel through the control of the attempt probabilities [4]. The power control game studies the choice of transmission power over a collision channel [6]. Finally, in congestion control the HD game can be used to study the choice between versions of TCP (transmission control protocols) to be used over the Internet [7].

In the HD game, there are two types of individuals: aggressive (Hawk, denoted by $\mathrm{H}$ ) and peaceful (Dove, denoted by D). In the MAC problem, the aggressive behavior corresponds to a high attempt rate. In power control a Hawk coincides with transmission at a high power, and in the congestion control it is the choice of an aggressive version of TCP (e.g. scalable TCP or high-speed TCP).

In this paper, we revisit the power control problem. We use the classical framework of evolutionary games, which we extend to a semi-dynamic context (see below). This framework deals with large populations in which individuals interact with each other through many local interactions, each interaction involving two randomly selected individuals. This pairwise interaction paradigm is relevant for sparse mobile networks in which one may neglect the possibility of simultaneous interference of more than two mobiles.

The standard HD game predicts when one type of behavior ( $\mathrm{H}$ or $\mathrm{D})$ would dominate in the long run, and when we may expect the coexistence of aggressive and peaceful individuals. The equilibrium fraction of each type of behavior is obtained by solving a 2 player auxiliary matrix game.
Several authors have studied dynamic variants of this game where individuals are characterized by their energy state [6], [7], [9]-[11]. A biological variant of this game can be found in [13]. Aggressive behavior requires more energy (which is the case in both the MAC problem as well as in the original HD example). The energy reserve of an individual is defined as the individual state. Thus actions of an individual influence not only the immediate fitness but also the future state of the individual. Individuals aim to maximize the total expected fitness during their lifetime. In these dynamic versions of the HD game, the individual strategy is no more a single choice between $\mathrm{H}$ and $\mathrm{D}$, but rather a collection of choices that prescribes how an individual should behave at each possible state.

We consider in this paper a semi-dynamic framework which inherits features from the static and dynamic frameworks. As in the dynamic setting, each player has an individual energy state and the player's action determines not only the immediate fitness but also the future state distribution. Yet in contrast to the dynamic versions of the game, we assume that an individual makes state independent choices. The individual chooses $i$ (where $i$ is $H$ or $D$ ) and once the choice is made, the same action $i$ is always used by this individual at any state. The individual is either always aggressive or always peaceful. The problem thus resembles the static framework in the fact that the individual has to choose only once, between $\mathrm{D}$ and $\mathrm{H}$.

We shall use the two central concepts of evolutionary games. The first is the concept of an Evolutionary Stable Strategy (ESS), which is a distribution of (deterministic or mixed) actions such that, if used, the population is immune against penetration of mutations. This notion is stronger than that of Nash equilibrium as ESS is robust against a deviation of a whole fraction of the population whereas the Nash equilibrium is defined with respect to possible deviations of a single player.

\section{MODEL}

Consider a sparse network that consists of a large population of mobile stations (MS). Apart from mobile stations, there are also many fix receivers: throw boxes, relays or base stations which are referred to as base stations (BS) in the remainder. We focus on the case where MSs only transmit when they are in the transmission range of a BS. That is, the situation in which mobiles themselves forward packets 
of other mobiles is explicitly excluded. As multiple MSs may transmit simultaneously to a BS, interference cannot be avoided. However, assuming that the network is sparse, we do not consider interference between multiple mobiles. That is, if interference occurs, at most two mobile terminals are involved, and the probability of interference between multiple mobile stations is neglected.

This brings us to the pairwise interaction paradigm of evolutionary games. It is assumed that interactions between individuals occur by some random selection process in which pairs of individuals are selected independently. We consider two types of terminals: one type transmits at high power while the other type transmits at low power. We refer to these by hawks $(\mathrm{H})$ and doves (D), respectively, thereby referring to the HD game. A mobile user (player) decides which terminal to use, and once this choice is made, he sticks to that choice of terminal for some predetermined time $T$. Considering only pairwise interaction, it is assumed that the sequence of types of terminals with which a given terminal interacts constitutes a sequence of i.i.d. random variables.

We consider two distinct models.

- Model 1 (MI): $T$ is some fixed large time, for example two years, which is approximately the expected time until one changes his/her cellular phone. We assume that $T$ does not depend on the type of the phone (H or D).

- Model 2 (M2): $T$ is the time until the mobile runs out of battery power. Note that in this case, $T$ is a function of the choice of type of battery. Indeed, as $\mathrm{H}$ consumes more energy than $D$, it will drain faster.

Models 1 and 2 above have interesting mathematical properties that guarantee the existence of an ESS as described at the end of Section IV and which in turn facilitate its computation.

The interference model is characterized by the probabilities for successful transmissions whereas the energy model at hand is characterized by the transition probabilities for the energy levels. We make the following assumptions.

Success probability: Consider a packet transmission of a terminal and let $\delta$ denote the probability that no other terminal interferes with its transmission. If this is not the case, there is interference between two terminals and the probability that the packet is transmitted successfully is determined by the types of the terminals involved. Let $p_{s}(i, j)$ denote the success probability of the first terminal assuming that this terminal plays $i$ while the other plays $j(i, j \in\{H, D\})$. We have,

$$
p_{s}(i, j)= \begin{cases}0 & \text { for }(i, j)=(D, H), \\ p_{1} & \text { for }(i, j)=(D, D), \\ 1 & \text { for }(i, j)=(H, D), \\ p_{2} & \text { for }(i, j)=(H, H) .\end{cases}
$$

Transition probabilities: If the energy level of an individual is $n$ and its action is $D$, then the energy level decreases to $n-1$ with probability $q_{1}$ or it remains unchanged with probability $q_{2}$. We assume $q_{1}+q_{2} \leq 1$. We do allow $q_{1}+q_{2}<1$, in which case we assume that there is a positive probability of $1-q_{1}-q_{2}$ for a breakdown which does not depend on the energy level. A breakdown is represented as a transition to an energy level zero.

Analogously, if the energy level of an individual is $n$ and its action is $H$, then the energy level decreases to $n-1$ with probability $q_{3}$ or it remains unchanged with probability $q_{4}$. Of course we have $q_{1}<q_{3}$. As for $\mathrm{D}$, we again allow that $q_{3}+$ $q_{4}<1$ in which case we shall have a breakdown probability $1-q_{3}-q_{4}$, a breakdown corresponding to a transition to energy level 0 .

Initial energy level: In the remainder, we assume that a mobile starts at energy level $N_{D}$ or $N_{H}$, depending on the type of mobile. The energy level represents the number of transmissions the mobile can do. As transmission by a hawk requires more energy, a dove will be able to transmit more times with the same battery: $N_{D}>N_{H}$. Finally, when the battery is empty, it is immediately replaced.

\section{PROPERTIES OF THE FITNESS}

Both hawks and doves aim to optimize the amount of data that can be send during the lifetime of the battery, hence the fitness is defined as follows

Definition 1. The long term fitness of a mobile is defined as the sum of the expected number of packets sent by that mobile during the lifetime of its battery. We denote by $V(j, i)$ the long term fitness of a mobile, given that it is of type $j$, and that all others are of type $i$, with $i, j \in\{H, D\}$.

Definition 2. Assume that at any time, a fraction $\alpha$ of the mobiles use action $D$, and the rest use $H$. We then denote by $V(j, \alpha)$ the corresponding long term fitness given that the mobile uses $j$. Moreover, let

$$
V(\beta, \alpha)=\beta V(D, \alpha)+(1-\beta) V(H, \alpha)
$$

be the fitness of a terminal that chooses mobile type $D$ (and always uses it) with probability $\beta$, and otherwise chooses type $D$ (with probability $1-\beta$ ).

\section{Evolutionary Stable Strategies}

\section{A. Nash equilibrium}

As usual, a symmetric strategy $\alpha$ is a Nash equilibrium if no player can do strictly better by a unilateral deviation to some other pure or mixed action $\beta$.

- For $i=H$ or $i=D, i$ is a pure Nash equilibrium if $V(i, i) \geq V(j, i)$ for $j=H, D$.

- Assuming model M1, we have that $\alpha$ is a mixed Nash equilibrium if $V(\alpha, \alpha) \geq V(\beta, \alpha)$ for all $\beta$.

- Assuming model M2, we have that $\alpha$ is a mixed Nash equilibrium if $V(\alpha, g(\alpha)) \geq V(\beta, g(\alpha))$ for all $\beta$, where $g$ is defined as,

$$
g(\alpha)=\frac{\alpha T_{D}}{\alpha T_{D}+(1-\alpha) T_{H}},
$$

with $T_{i}$ the time that a battery of a type $i$ mobile empties. An equilibrium is said to be strict if any deviation by any player results in a strictly worse fitness for that player. 


\section{B. Definition of a standard evolutionary game}

Suppose that the whole population uses a strategy $q$ and that a small fraction $\epsilon$ (called "mutations") adopts another strategy $p$. Evolutionary forces are expected to select against $p$ if

$$
V(q, \epsilon p+(1-\epsilon) q)>V(p, \epsilon p+(1-\epsilon) q)
$$

Definition 3. A strategy $q$ is said to be an Evolutionary Stable Strategy (ESS) if for every $p \neq q$ there exists some $\bar{\epsilon}_{y}>0$ such that (3) holds for all $\epsilon \in\left(0, \bar{\epsilon}_{y}\right)$.

We shall make use of the following characterization of an ESS [12]:

Theorem 1. A strategy $q$ is an Evolutionary Stable Strategy if and only if $\forall p \neq q$ the following conditions holds:

$$
V(q, q) \geq V(p, q),
$$

and if

$$
V(q, q)=V(p, q) \text { then } V(q, p)>V(p, p) .
$$

The first condition says that the ESS is a Nash equilibrium in the game that describes the interaction between two players. Conversely, if $q$ is a strict Nash equilibrium in that game then it is an ESS in the evolutionary game.

The second condition, referred to as "Maynard Smith's second condition", states that if $q$ is a Nash equilibrium but not a strict Nash equilibrium (i.e. the fitness of a deviation $p$ from $q$ does as good as $q$ when the rest of the population uses $q$ ), then $q$ can still be an ESS if it has an advantage in that it can invade the mutants strategy $p$. In other words, in a population where every one uses $p$, a small deviation to $q$ does strictly better than everyone using $p$.

We shall consider evolutionary games where each player has a finite number of available pure actions and where the set of strategies of a player is the set of probability distributions over his actions. Let $V(p, q)$ denote the expected fitness (utility) for a player when playing a mixed policy $p$ and when the fraction of the population that plays each pure strategy $i$ is given by $q(i)$. The expected fitness is then linear in both $p$ and $q$ and can be written as $p \mathbf{V} q^{T}$ where $\mathbf{V}$ is the matrix whose $i, j$ th entry equals $V(i, j)$, and where $p$ (resp. $q$ ) is a row vector whose $i$ th entry is $p(i)$ (resp. $q(i)$ ). Theorem 1 then states that the ESS of an evolutionary game can be characterized by properties of the equilibria of an auxiliary game. In our case this auxiliary game is the matrix game $\mathbf{V}$. Note that not every matrix game has an ESS.

\section{ESS IN THE SEMI-DYNAMIC GAME}

Consider the following two pure strategies of a player: (i) always play $\mathrm{D}$, and (ii) always play $\mathrm{H}$. With some abuse of notation we denote these policies by $\mathrm{D}$ and $\mathrm{H}$. When writing the long term fitness of players as a function of the system parameters, we shall see that the fitness is linear in $p$ and $q$ whereby $p$ are now probabilities over the strategies $H$ and $D$ and not over the actions $H$ and $D$. This means that a mixed strategy is obtained by tossing a coin, and according to the outcome, the player always uses $D$ or always uses $H$. Notice that if we choose between action $D$ and $H$ with some probability $q$ at each time instant, then the expected fitness need not be linear in $q$. This bilinear form of semidynamic games allows us to apply directly the standard theory of evolutionary games to the semi-dynamic case.

Recall that, even though we assume that each individual mobile $j$ always plays the same action, the sequence of actions that are played by the mobiles encountered by some tagged mobile are i.i.d.

While working with mixed strategies allows for directly applying much of the framework of standard evolutionary games, these policies do not allow for an evolution, as once we perform the initial randomized selection between $D$ and $H$, we shall always stick to that choice. Hence, to combine both the flexibility that allows for evolution together with the linear properties of the auxiliary game (the matrix game introduced above), we assume that each mobile uses mixed policies for some limited time $T$, after which a new choice is made and so on.

\section{COMPUTING THE EQUILIBRIUM}

Let $V_{n}(i, \alpha)$ denotes the expected fitness of a user who plays $i$ and starts at energy level $n, i, j \in\{H, D\}$. In view of this definition we have, $V(D, \alpha)=V_{N_{D}}(D, \alpha)$ and $V(H, \alpha)=$ $V_{N_{H}}(H, \alpha)$. We find the following recursions for $V_{n}(i, \alpha)$,

$$
\begin{aligned}
V_{n}(D, \alpha)= & \left(\delta+(1-\delta) \alpha p_{1}\right)+q_{1} V_{n-1}(D, \alpha) \\
& +q_{2} V_{n}(D, \alpha) \\
V_{n}(H, \alpha)= & (\delta+(1-\delta) \alpha)+(1-\alpha)(1-\delta) p_{2} \\
& +q_{3} V_{n-1}(H, \alpha)+q_{4} V_{n}(H, \alpha) .
\end{aligned}
$$

The first equation expresses the total expected fitness of a mobile of type $\mathrm{D}$ when starting with $n$ units of energy, till its battery empties. Hence, the equation is composed of two expressions:

(i) The expected fitness corresponding to the current transmission: with probability $\delta$ there is no interference at all so the fitness is one unit. With probability $(1-\delta)$ there is an interaction with another mobile. The fitness equals $p_{1}$ when both mobiles use $D$ which occurs with probability $\alpha$. Otherwise, it is zero.

(ii) The expected fitness collected after the transmission: we first note that with probability $q_{1}$, the energy level after the transmission equals $n-1$, so the expected fitness to go is $q_{1} V_{n-1}(D, \alpha)$. With probability $q_{2}$ the energy level is unchanged so the expected fitness collected after the transmission is $q_{2} V_{n}(D, \alpha)$.

The second equation can be explained following similar lines. 
Solving the recursions for $q_{1}+q_{2}<1$ and $q_{3}+q_{4}<1$ yields,

$$
\begin{aligned}
V_{n}(D, \alpha) & =\frac{\delta+\alpha p_{1} \bar{\delta}}{1-q_{1}-q_{2}}\left(1-\left(\frac{q_{1}}{1-q_{2}}\right)^{n}\right), \\
V_{n}(H, \alpha) & =\frac{\delta+\alpha \bar{\delta}+p_{2} \bar{\delta}(1-\alpha)}{1-q_{3}-q_{4}}\left(1-\left(\frac{q_{3}}{1-q_{4}}\right)^{n}\right),
\end{aligned}
$$

with $\bar{\delta}=1-\delta$ and whereby we assumed $V_{0}(D, \alpha)=$ $V_{0}(H, \alpha)=0$. That is, no fitness can be collected if the battery is empty.

\section{A. With Breakdowns}

Lemma 1. Assume that both hawks and doves are subjected to breakdowns $\left(q_{1}+q_{2} \neq 1\right.$ and $\left.q_{3}+q_{4} \neq 1\right)$, we have for $i \in\{D, H\}$,

$$
V(i, \alpha)=\alpha V(i, D)+(1-\alpha) V(i, H),
$$

with,

$$
\begin{aligned}
V(D, D) & =\frac{\delta+p_{1}(1-\delta)}{1-q_{1}-q_{2}}\left(\frac{q_{1}}{1-q_{2}}\right)^{N_{D}} \\
V(H, D) & =\frac{1}{1-q_{3}-q_{4}}\left(1-\left(\frac{q_{3}}{1-q_{4}}\right)^{N_{H}}\right), \\
V(H, H) & =\frac{\delta+p_{2}(1-\delta)}{1-q_{3}-q_{4}}\left(1-\left(\frac{q_{3}}{1-q_{4}}\right)^{N_{H}}\right), \\
V(D, H) & =\frac{\delta}{1-q_{1}-q_{2}}\left(\frac{q_{1}}{1-q_{2}}\right)^{N_{D}} .
\end{aligned}
$$

This allows us to express the equilibrium as follows.

Corollary 1. Assuming non-zero breakdown probability, the following holds.

(i) $D$ is a pure equilibrium if

$$
\frac{1}{1-q_{3}-q_{4}} \cdot\left(\frac{q_{3}}{1-q_{4}}\right)^{N_{H}}+\frac{\delta+p_{1}(1-\delta)}{1-q_{1}-q_{2}} \cdot\left(\frac{q_{1}}{1-q_{2}}\right)^{N_{D}}>\frac{1}{1-q_{3}-q_{4}}
$$

(ii) $H$ is a pure equilibrium if

$$
\frac{\delta+p_{2}(1-\delta)}{1-q_{3}-q_{4}} \cdot\left(\frac{q_{3}}{1-q_{4}}\right)^{N_{H}}+\frac{\delta}{1-q_{1}-q_{2}} \cdot\left(\frac{q_{1}}{1-q_{2}}\right)^{N_{D}}<\frac{\delta+p_{2}(1-\delta)}{1-q_{3}-q_{4}}
$$

(iii) Let

$$
\alpha^{*}=\frac{\theta \cdot \frac{\delta}{1-q_{1}-q_{2}}-\rho \cdot \frac{\left(\delta+p_{2}(1-\delta)\right)}{1-q_{3}-q_{4}}}{\theta \cdot \frac{\left((1-\delta)\left(1-p_{2}\right)\right)}{1-q_{3}-q_{4}}-\rho \cdot \frac{\left((1-\delta) p_{1}\right)}{1-q_{1}-q_{2}}}
$$

where $\theta=\left(1-\left(\frac{q_{1}}{1-q_{2}}\right)^{N D}\right)$ and $\rho=$ $\left(1-\left(\frac{q_{1}}{1-q_{2}}\right)^{N H}\right)$. If $\alpha^{*}$ is in the interior of the unit interval then it is a mixed ESS.
Notice that the existence of the mixed strategy $\alpha^{*}$ is still not assured. Indeed, one must identify conditions on the parameters in order to guarantee that $\alpha^{*}$ lies in between 0 and 1.

Lemma 2. The mixed ESS $\alpha^{*}$ is given by

$$
\alpha^{*}=\frac{V(H, H)-V(D, H)}{V(D, D)+V(H, H)-V(H, D)-V(D, H)}
$$

We have the following existence conditions

(i) $V(D, D)>V(H, D)$ and $V(H, H)>V(D, H)$ or

(ii) $V(D, D)>V(H, D), V(H, H)<V(D, H)$ and $|V(H, H)-V(D, H)|>|V(D, D)-V(H, D)|$.

We can therefore compute the value of the HD game.

Corollary 2. The value of the Hawk-Dove game is

$$
V=\frac{|\Delta|}{V(H, H)+V(D, D)-V(H, D)-V(D, H)}
$$

where $|\Delta|$ stands for the determinant of the matrix game $G$.

\section{B. Without Breakdowns}

Assume now that hawks and doves are not subjected to breakdowns. We then have $q_{1}=1-q_{2}$ and $q_{3}=1-q_{4}$, which yields

$$
\begin{aligned}
& V_{n}(D, \alpha)=\frac{\alpha p_{1}(1-\delta)+\delta}{q_{1}} n \\
& V_{n}(H, \alpha)=\frac{(1-\delta)(1-\alpha) p_{2}+\delta+\alpha(1-\delta)}{q_{3}} n
\end{aligned}
$$

In the latter case, we conclude the following

Lemma 3. In the absence of breakdowns $\left(q_{1}=1-q_{2}\right.$ and $\left.q_{3}=1-q_{4}\right)$, we have for $i \in\{D, H\}$,

$$
V(i, \alpha)=\alpha V(i, D)+(1-\alpha) V(i, H),
$$

with,

$$
\begin{array}{ll}
V(D, D)=\frac{p_{1}(1-\delta)+\delta}{q_{1}} N_{D}, & V(H, D)=\frac{1}{q_{3}} N_{H}, \\
V(H, H)=\frac{(1-\delta) p_{2}+\delta}{q_{3}} N_{H}, & V(D, H)=\frac{\delta}{q_{1}} N_{D}
\end{array}
$$

This gives us the following equilibria.

Corollary 3. In the absence of breakdowns, the following holds.

(i) $D$ is a pure equilibrium if $\left(p_{1}(1-\delta)+\delta\right) q_{3} N_{D}>q_{1} N_{H}$.

(ii) $H$ is a pure equilibrium if $\left(p_{2}(1-\delta)+\delta\right) q_{1} N_{H}>\delta q_{3} N_{D}$.

(iii) Let

$$
\alpha^{*}=\frac{\delta q_{3} N_{D}-q_{1}\left(p_{2}(1-\delta)+\delta\right) N_{H}}{(1-\delta)\left(q_{1}\left(1-p_{2}\right) N_{H}-q_{3} p_{1} N_{D}\right)}
$$




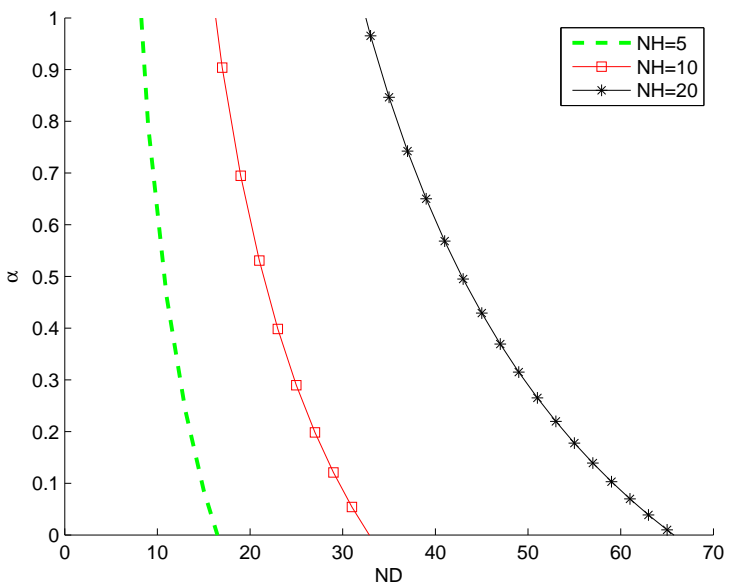

Fig. 1. Variation of $\alpha$ as function of $N_{D}$.

If $\alpha^{*}$ is in the interior of the unit interval then it is a mixed ESS.

At the equilibrium, Let $\underline{\beta}=(\beta, 1-\beta)$ and $\underline{\alpha}=(\alpha, 1-\alpha)$ be two row vectors of probability measures over the available actions $D, H$. Let $\mathbf{V}$ be a matrix whose $(i, j)$ th entry is given by $V(i, j)$. In accordance with Definition 2 , denote by $V(\beta, \alpha)$ the expected fitness of a player who always plays strategy $D$ with probability $\beta$ and always $H$ with probability $1-\beta$, while the fraction of individuals in the population that play $D$ is $\alpha$. We then make the following key observation regarding the total expected utility.

Lemma 4. The expected utility for a player that chooses to be $D$ with probability $\beta$ given that the fraction of $D$ in the population is $\alpha$ can be written in a vector form as

$$
V(\beta, \alpha)=\underline{\beta} \mathbf{V} \underline{\alpha}^{T} .
$$

It is thus bilinear. It can therefore be interpreted as the expected fitness for a player in an equivalent one shot game (a symmetric static evolutionary game) where the fraction of $D$ in the population is $\alpha$ and where the player chooses $D$ with probability $\beta$. The equilibrium given in Lemma 1 is an ESS.

\section{THE INITIAL ENERGY PARADOX}

Consider the case without recharging (the case of recharging is similar). Figure 1 shows the impact of the initial energy level - say $N_{D}$ - on the equilibrium proportion $\alpha$ of $D$. We here used the following variables: $p_{1}=0.3, p_{2}=0.8, q_{1}=0.6$, $q_{3}=0.9, \delta=0.1$ and $N_{H}=1$. We can now identify a surprising paradox in the HD game referred to as the initial energy paradox. We see that the larger $N_{D}$ is, the smaller is the fraction $\alpha$. This is paradoxical because a larger $N_{D}$ gives an advantage to the Doves - by choosing $D$ a mobile can transmit more packets: both $V(D, D)$ and $V(D, H)$ increase. As usual in paradoxes in games, the equilibrium is not necessarily monotone increasing in the utilities. Some intuition to this behavior is obtained by recalling that a mixed equilibrium $\alpha^{*}$ is characterized by the indifference principle that states that $\alpha^{*}$ is such that the fitness of a player is the same under $D$ and $H$. Now, by changing $N_{D}$ this does not change the fitness of $H$. It increases, but $V(D, H)$ increases more than $V(D, D)$. Therefore to keep each player indifferent between $D$ and $H$, $\alpha^{*}$ should decrease!

\section{CONCLUSION}

We have studied in this paper a semi-dynamic version of the Hawk and Dove game within the framework of evolutionary games. We have considered the property that the state transition of an individual player depend only on its own action and not on the behavior of other players that it meets. The action of the other player has only an impact on the immediate fitness. We identify a surprising paradox in our Hawk-Dove game namely, the initial energy paradox which offers insights on how mobiles behave in the framework of semi-dynamic Hawk and Dove game.

\section{REFERENCES}

[1] D. J. Goodman and N. B. Mandayam, "Power control for wireless data", IEEE Personal Commun., vol. 7, pp. 48-54, 2000.

[2] D. Famolari, N. Mandayam, D. Goodman, and V. Shah, "A new framework for power control in wireless data networks: Games, utility, and pricing", in Wireless Multimedia Network Technologies. New York: Springer, 1999.

[3] J. Maynard Smith, Game Theory and the Evolution of Fighting In John Maynard Smith, On Evolution (Edinburgh: Edinburgh University Press), pp.8-28, 1972.

[4] H. Tembine, E. Altman, and R. El-Azouzi. "Delayed Evolutionary Game Dynamics applied to the Medium Access Control", Bionetworks 2007, Pisa, Italy.

[5] P. Wiecek, E. Altman and Y. Hayel, "Stochastic state dependent population games in wirelesss communications", Transactions on Autoatic Control, Volume 56 Number 3, pages 492-505, March, 2011.

[6] Yezekael Hayel, Hamidou Tembine, Eitan Altman, Rachid El-Azouzi "A Markov Decision Evolutionary Game for Individual Energy Management", Annals of the International Society of Dynamic Games, 2009.

[7] E. Altman, R. El-Azouzi, Y. Hayel and H. Tembine, "The evolution of transport protocols: An evolutionary game perspective", Computer Networks (Elsevier) Volume 53, Issue 10, 14 July 2009, pp 1751-1759.

[8] J. Maynard Smith, Evolution and the Theory of Games, Cambridge University Press, 1982.

[9] E. Altman, Y. Hayel, H. Tembine and R. El-Azouzi Markov Decision Evolutionary Games with Expected Average Fitness, Evolutionary Ecology Research, 11 (4), pp. 677-689, 2009.

[10] E. Altman and Y. Hayel, "Markov Decision Evolutionary Games", IEEE Transactions on Automatic Control, VOL 55 Issue 6, June 2010.

[11] J. McNamara, S. Merad, E. Collins, The Hawk-Dove Game as an Average-Cost Problem, in Avdances in Applied Probability, Vol. 23, no. 4, pp. 667-682, 1991.

[12] M. van Veelen and P. Spreij. Evolution in games with a continuous action space. Economic Theory, 39(3):355-376, 2008.

[13] E. Altman, J. Gaillard, M. Haddad and P. Wiecek, "Dynamic Hawk and Dove games within flocks of birds", Bionetics, York, England, 2011. 